\title{
Multi-Fidelity Machine Learning Models for Accurate Bandgap Predictions of Solids
}

\author{
G. Pilania, ${ }^{1}$ J. E. Gubernatis, ${ }^{2}$ and T. Lookman $^{2}$ \\ ${ }^{1}$ Materials Science and Technology Division, Los Alamos National Laboratory, Los Alamos, New Mexico 87545, USA \\ ${ }^{2}$ Theoretical Division, Los Alamos National Laboratory, Los Alamos 87545, NM, USA
}

(Dated: November 7, 2016)

\begin{abstract}
We present a multi-fidelity co-kriging statistical learning framework that combines variable-fidelity quantum mechanical calculations of bandgaps to generate a machine-learned model that enables low-cost accurate predictions of the bandgaps at the highest fidelity level. In addition, the adopted Gaussian process regression formulation allows us to predict the underlying uncertainties as a measure of our confidence in the predictions. Using a set of 600 elpasolite compounds as an example dataset and using semi-local and hybrid exchange correlation functionals within density functional theory as two levels of fidelities, we demonstrate the excellent learning performance of the method against actual high fidelity quantum mechanical calculations of the bandgaps. The presented statistical learning method is not restricted to bandgaps or electronic structure methods and extends the utility of high throughput property predictions in a significant way.
\end{abstract}

\section{INTRODUCTION}

Owing to its central role in modern device physics, energy harvesting, energy storage, catalysis and other technologically relevant applications,${ }^{1}$ the bandgap often serves as a crucial screening parameter in rational design of functional materials. ${ }^{2-4}$ As available experimental data for bandgaps is generally limited, ${ }^{5,6}$ it is not surprising that a number of recent high throughput chemical space explorations, in search of compounds with improved functionalities, have targeted the calculation of the bandgap. ${ }^{7-16}$

Given that accurate calculations of bandgaps are time consuming and resource intensive, we demonstrate in this paper the use of a statistical learning method that generates machine-learned models to obviate the costs of such calculations. The multi-fidelity method presented combines many inexpensive lower accuracy computations of bandgaps with fewer expensive higher accuracy computations to predict bandgaps whose accuracies are comparable to those produced by the higher accuracy calculations alone. The greater the difference in the costs of the calculations, the greater is the cost advantage of the method we demonstrate. Because a natural and well documented accuracy hierarchy exists for bandgap calculation methods, bandgap computations are natural for the method presented. In principle, the method can be applied to the predictions of computed or experimental properties where the data is grouped into different levels of accuracy.

Our approach takes available bandgap prediction methods from different levels of theories with different fidelities to estimate bandgaps at the fidelity level of the more accurate and computationally expensive theory. On one end of the fidelity spectrum, we may have have surrogate models, perhaps obtained via high throughput computations, but less trustworthy or known to be inaccurate. On the other end we may have high-fidelity models that enable quite accurate estimates. In the present context of quantum mechanical computations of bandgaps, the variable fidelity can be thought of as bandgaps computed from different levels of exchange correlation functionals within DFT, ${ }^{17-20}$ as creatively captured in Jacob's ladder of density functional approximations put forward by Perdew (Fig. 1$)^{21}$ as well as expressed by the spectrum of beyond-DFT approaches such as the GW method, ${ }^{22,23}$ Møller-Plesset perturbation theory (MP2), ${ }^{24}$ and configuration interaction $(\mathrm{CI}) .^{25}$

We use the statistical learning approach of multifidelity co-kriging on a data set of 640 double perovskite halide compounds for which the bandgap energies can be calculated with two levels of fidelity (the lower fidelity Perdew-Burke-Ernzerhoff $(\mathrm{PBE})^{19}$ and higher fidelity Heyd-Scuseria-Ernzerhof (HSE06) ${ }^{17}$ exchangecorrelation functional approximations). We make predictions of the HSE06 bangap energies by considering different numbers of the PBE bandgaps in the combined data in which the number of high fidelity bandgaps is a subset of the low fidelity ones. One of our central conclusions is not surprisingly that the accuracy of the prediction increases as the number of high fidelity bandgaps increases in the training set. Another of our conclusion is more importantly that the accuracy of the predictions increases as the number of low fidelity data increases in the training set. We deduce these conclusions from heat maps of the mean square error in the HSE06 predictions, made by our trained learning model on unseen data, as a function of the number of low fidelity data points and the relative proportion of high fidelity data used.

Our approach is markedly different from other multifidelity approaches in the literature which are based on using low fidelity data (e.g., PBE bandgaps) as features in the machine learning (ML) model ${ }^{26}$ and therefore strictly require low fidelity data for all materials for which predictions are to be made using the trained model. This can be particularly challenging and extremely computationally demanding when faced with a combinatorial problem that targets to explore a vast chemical and configurational space. Here, we present a framework for a multi-fidelity Gaussian process (GP) based ML regression model that seamlessly combines bandgap inputs 


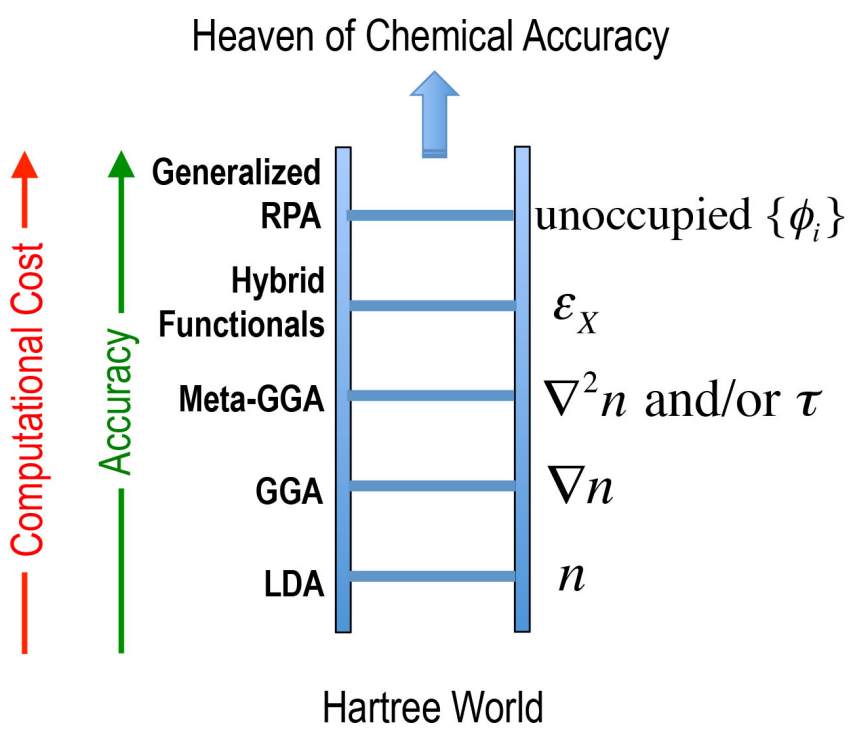

FIG. 1. Jacob's ladder of density functional approximations to the exchange-correlation energy (as put forward by Perdew, adapted from Ref. [21]) presents a prototypical example of multi-fidelity computations in materials properties, where a natural hierarchy exists in both computational cost and accuracy. The present study demonstrates the effectiveness of multi-fidelity ML approach for a bandgap dataset of elpasolite compounds computed at the GGA and the hybrid functional levels. Symbols $n, \epsilon_{x}$ and $\phi_{i}$ in the figure represent the ground state charge density, the exact exchange and Kohn-Sham orbitals, respectively.

from two or more levels of fidelities to make accurate predictions of the bandgaps for the highest fidelity. Furthermore, adopting a nested setting for variable-fidelity training data, the model requires high-fidelity training data only on a subset of compounds for which low-fidelity training data is readily available. More importantly, the trained model can make efficient yet accurate predictions for the highest-fidelity bandgaps even in the absence of the low-fidelity bandgap data for the prediction set compounds. In addition to the bandgap predictions obtained with lower cost, the adopted GP-regression framework also allows us to predict the underlying uncertainties as a measure of our confidence in the predictions.

The outline of our paper is as follows. In Sec. II we review multi-fidelity approaches as they have been studied in the engineering context and DFT methods. We also introduce and discuss the co-kriging approach which we utilize in this study. This introduction is followed by a description of the data set of double perovskites and the features used to the train the Gaussian model used in co-kriging. We also provide the computational details underpinning our DFT calculations. Section III presents and discusses the results of our calculations as we show how our predictions of the bandgaps are successively refined depending on the data sizes of the low and high fidelity data. Finally, Sec. IV concludes and discusses the significance and implications of our work.

\section{BACKGROUND AND METHODOLOGY}

\section{A. Motivation and Past Work}

Fueled by recent advances in methodologies and computational power, density functional theory (DFT) has become a standard workhorse for ab initio electronic structure calculations, providing the best tradeoff between predictive accuracy and computational efficiency. ${ }^{27}$ While the standard implementations of DFT are widely employed to compute structural, electronic, electrical, magnetic and other properties of plethora of materials, they suffer from a well known deficiency (also known as the "bandgap problem") ${ }^{28}$ in which DFT within local or semi-local exchange-correlation functionals fails to correctly predict the energy gaps between occupied and unoccupied states. In fact, the experimental bandgap $\epsilon_{\text {exp }}$ is often severely underestimated by the Kohn-Sham gap $\epsilon_{K S}$. This underestimation is attributed to the inherent lack of derivative discontinuity ${ }^{29}$ and delocalization error ${ }^{30,31}$ within the local or semi-local exchange-correlation functionals such as the local density approximation (LDA) or the generalized gradient approximation (GGA). ${ }^{32}$

For the exact Kohn-Sham formalism, the physical gap equals $\epsilon_{K S}$ plus the derivative discontinuity of the exchange-correlation (xc) energy with respect to the number of electrons. ${ }^{29,33,34}$ In a non-exact KS approach with an approximate xc energy functional, the above relation may not be exact and, in addition, the approximate functional may not reproduce the correct xc energy derivatives. ${ }^{30,31}$ Correction techniques such as the DFT $+\mathrm{U}$ can improve the KS gap only to a limited extent. ${ }^{35}$ Recently developed more advanced exchange-correlation functionals, such as the modified Becke-Johnson (mBJ) functional by Tran and Blaha ${ }^{36}$ and strongly constrained and appropriately normed $(\mathrm{SCAN})^{37}$ meta-GGA certainly improve over the classic LDA and GGA functionals, but do not completely alleviate the problem. Several other alternative approaches are frequently employed to address the bandgap problem, including the delta self-consistent-field $(\Delta-\mathrm{SCF})$ method, ${ }^{38}$ hybrid functional methods ${ }^{17}$ and the quasiparticle GW calculations based on the many-body perturbation theory. ${ }^{39} \Delta$-SCF formalism requires the evaluation of the total energy at three different numbers of electrons $(\mathrm{N}, \mathrm{N}+\delta$, and $\mathrm{N}-\delta), \mathrm{N}$ being the number of electrons in the neutral ground state. $\delta$-representing the amount of charge added or removed to simulate the excitation process - depends on $\mathrm{N}$ and is determined empirically to best yield the experimental bandgap. While the $\Delta$-SCF method is not entirely parameter-free, the latter two approaches - although capable of providing bandgap estimates in good agreement with the corresponding experimental measurements - suffer from high computational costs.

ML methods have recently had phenomenal success in condensed matter physics and materials science for 
high throughput screening and accelerated property predictions. ${ }^{40-42}$ Even with a limited set of prior data to train on, ML-based approaches have been successfully employed, for instance, to accurately estimate a wide range of material properties for molecular ${ }^{43,44}$ and periodic systems, ${ }^{7,45}$ to develop adaptive force fields, ${ }^{46,47}$ to devise schemes for crystal structure classifications, ${ }^{48-50}$ to predict dielectric breakdown strength of insulators ${ }^{51,52}$ and to come up with alternative self-consistent solutions for quantum mechanics. ${ }^{53}$

Techniques of statistical learning have also presented an alternative and potential route towards addressing the aforementioned bandgap problem. Some past efforts in this direction used regression (learning) methods such as ordinary least squares regression (OLSR), ${ }^{15,26,54}$ sparse partial least square regression, ${ }^{54}$ least absolute shrinkage and selection operator (LASSO), ${ }^{26,54}$ support vector regression (SVR), ${ }^{26,55}$ artificial neural networks $(\mathrm{ANNs})^{55,56}$ and kernel ridge regression $(\mathrm{KRR})^{57,58}$ to predict either experimental bandgaps or bandgaps from computationally expansive beyond-DFT methods using DFT-KS bandgaps and properties of constitutive elements such as atomic valence, atomic number, melting points of elemental standard states, electronegativity, first atomic ionization potential, pseudopotential radii, etc. as features.

The past work shows that while the ML models based solely on the features of the constitutive elements often lead to limited accuracy and generalizability, the models that use local or semi-local KS-bandgaps to predict bandgaps from a higher level of theory (such as hybridfunctionals or the GW method) or experiments, though accurate, are unsuitable for making high throughput predictions on vast chemical spaces since they require the local or semi-local KS-bandgaps for the entire set of target compounds as input to the make predictions. ${ }^{26,57}$

\section{B. Multi-fidelity Co-kriging Regression Model}

In this section, we summarize the co-kriging regression method that we employed to make accurate predictions of the high-fidelity HSE bandgaps. Co-kriging regression is an extension of the well known kriging method to situations where model outputs of different levels of fidelity (i.e., variations both in computational cost and accuracy) are available to learn a property of interest. The approach we used was established by Kennedy and O'Hagan, ${ }^{59}$ who put forward a coherent mathematical framework for blending heterogeneous variablefidelity information sources, creating a natural setting for multi-fidelity modeling. Subsequently, Forrester et al. ${ }^{60}$ adopted this framework to build a two-level co-kriging scheme, discussed the details of the estimation of model parameters and successfully demonstrated its application in an optimization scheme.

While the auto-regressive scheme of Kennedy and O'Hagan ${ }^{59}$ is quite general in the sense that it can be applied to a situation where $s$-levels of variable-fidelity estimates are available, it suffers from practical limitations pertaining to computational efficiency when the number of levels $s$ or number of data points grow large. A significant breakthrough was achieved by recent work of Le Gratiet and Garnier, ${ }^{61,62}$ who proved that any co-kriging scheme with $s$-levels of variable-fidelity information sources can be effectively decoupled, and equivalently reformulated in a recursive fashion as an $s$ independent kriging problem. This result allows for the construction of predictive co-kriging schemes by solving a sequence of simpler kriging problems that involve covariance matrices of smaller dimensions.

In the present work, we used a 2-level co-kriging model with the HSE06 and PBE bandgaps representing the high-fidelity (expensive and accurate) and low-fidelity (cheap and less trustworthy) estimates. Closely following the convention introduced by Forrester et al. ${ }^{60}$ we represent the $n_{e}$ data points for the HSE06 bandgaps as $\mathbf{y}_{\mathbf{e}}$ for a set of materials represented by a matrix $\mathbf{X}_{\mathbf{e}}$ and the $n_{c}$ data points of PBE bandgaps as $\mathbf{y}_{\mathbf{c}}$ for a set of materials represented by $\mathbf{X}_{\mathbf{c}}$. Here, $\mathbf{X}_{\mathbf{e}}$ is assumed to be a subset of $\mathbf{X}_{\mathbf{c}}$; i.e., $\mathbf{X}_{\mathbf{e}} \subset \mathbf{X}_{\mathbf{c}}$. These datasets are concatenated to give a combined set of points as:

$$
\begin{gathered}
\mathbf{X}=\left(\begin{array}{l}
\mathbf{X}_{\mathbf{c}} \\
\mathbf{X}_{\mathbf{e}}
\end{array}\right)=\left(\mathbf{x}_{c}^{(1)}, \ldots, \mathbf{x}_{c}^{\left(n_{c}\right)}, \mathbf{x}_{e}^{(1)}, \ldots, \mathbf{x}_{e}^{\left(n_{e}\right)}\right)^{\mathrm{T}} \\
\mathbf{y}=\left(\begin{array}{l}
\mathbf{y}_{\mathbf{c}} \\
\mathbf{y}_{\mathbf{e}}
\end{array}\right)=\left(y_{c}^{(1)}, \ldots, y_{c}^{\left(n_{c}\right)}, y_{e}^{(1)}, \ldots, y_{e}^{\left(n_{e}\right)}\right)^{\mathrm{T}}
\end{gathered}
$$

Where, each of the $\mathbf{x}_{c}^{(i)}$ and $\mathbf{x}_{e}^{(i)}$ is a $d$-dimensional feature vector that uniquely fingerprints the material compositions in a target chemical space. Furthermore, in a close analogy with kriging, co-kriging also assumes that the value at a point in $\mathbf{X}$ is a realization of a Gaussian random variable. Therefore, we also have a random field

$$
\begin{aligned}
\mathbf{Z} & =\left(\begin{array}{l}
\mathbf{Z}_{\mathbf{c}}\left(\mathbf{X}_{\mathbf{c}}\right) \\
\mathbf{Z}_{\mathbf{e}}\left(\mathbf{X}_{\mathbf{e}}\right)
\end{array}\right) \\
& =\left(Z_{c}\left(\mathbf{x}_{c}^{(1)}\right), \ldots, Z_{c}\left(\mathbf{x}_{c}^{\left(n_{c}\right)}\right), Z_{e}\left(\mathbf{x}_{e}^{(1)}\right), \ldots, Z_{e}\left(\mathbf{x}_{e}^{\left(n_{e}\right)}\right)\right)^{\mathrm{T}} .
\end{aligned}
$$

The autoregressive scheme of Kennedy and O'Hagan ${ }^{59}$ writes the high-fidelity output as the low-fidelity output multiplied by a scaling factor $\rho$ plus an another independent Gaussian process $Z_{d}$ (i.e., $\left.Z_{c}(\mathbf{x}) \perp Z_{d}(\mathbf{x})\right)$ which represents the difference between $\rho Z_{c}$ and $Z_{e}$, as follows:

$$
Z_{e}(\mathbf{x})=\rho Z_{c}(\mathbf{x})+Z_{d}(\mathbf{x}) .
$$

This model is based on the Markov property introduced by Kennedy and O'Hagan that assumes if given $Z_{c}(\mathbf{x})$, we can learn nothing more about $Z_{e}(\mathbf{x})$ from any other model output $Z_{c}\left(\mathbf{x}^{\prime}\right)$, for $\mathbf{x} \neq \mathbf{x}^{\prime}$; i.e.,

$$
\operatorname{Cov}\left(Z_{e}(\mathbf{x}), Z_{c}\left(\mathbf{x}^{\prime}\right) \mid Z_{c}(\mathbf{x})=0 \quad \forall \mathbf{x} \neq \mathbf{x}^{\prime}\right.
$$


To describe the dependence structure of the Gaussian process $Z_{c}(\cdot)$ and $Z_{d}(\cdot)$, we consider a squared exponential kernel of the form $k\left(\mathbf{x}, \mathbf{x}^{\prime}\right)=\sigma^{2} \Im\left(\mathbf{x}, \mathbf{x}^{\prime}, \boldsymbol{\theta}\right)$, parametrized with the vector $\boldsymbol{\theta}$ and the variance parameter $\sigma^{2}$. The correlations between two points, represented by the $d$-dimensional feature vectors, $\mathbf{x}$ and $\mathbf{x}^{\prime}$ are captured by $\Im\left(\mathbf{x}, \mathbf{x}^{\prime}, \boldsymbol{\theta}\right)$, as:

$$
\mathbf{K}=\left(\begin{array}{ccc}
\operatorname{cov}\left\{Z_{c}\left(\mathbf{X}_{\mathbf{c}}\right), Z_{c}\left(\mathbf{X}_{\mathbf{c}}\right)\right\} & \operatorname{cov}\left\{Z_{e}\left(\mathbf{X}_{\mathbf{e}}\right), Z_{c}\left(\mathbf{X}_{\mathbf{c}}\right)\right\} \\
\operatorname{cov}\left\{Z_{e}\left(\mathbf{X}_{\mathbf{e}}\right), Z_{c}\left(\mathbf{X}_{\mathbf{c}}\right)\right\} & \operatorname{cov}\left\{Z_{e}\left(\mathbf{X}_{\mathbf{e}}\right), Z_{e}\left(\mathbf{X}_{\mathbf{e}}\right)\right\}
\end{array}\right)=\left(\begin{array}{cc}
\sigma_{c}^{2} \Im_{\mathbf{c}}\left(\mathbf{X}_{\mathbf{c}}, \mathbf{X}_{\mathbf{c}}\right) & \rho \sigma_{c}^{2} \Im_{\mathbf{c}}\left(\mathbf{X}_{\mathbf{c}}, \mathbf{X}_{\mathbf{e}}\right) \\
\rho \sigma_{c}^{2} \Im_{\mathbf{c}}\left(\mathbf{X}_{\mathbf{c}}, \mathbf{X}_{\mathbf{e}}\right) & \rho^{2} \sigma_{c}^{2} \Im_{\mathbf{c}}\left(\mathbf{X}_{\mathbf{e}}, \mathbf{X}_{\mathbf{e}}\right)+\sigma_{d}^{2} \Im_{\mathbf{d}}\left(\mathbf{X}_{\mathbf{e}}, \mathbf{X}_{\mathbf{e}}\right)
\end{array}\right)
$$

Note that since there are two correlations $\Im_{\mathbf{c}}$ and $\Im_{\mathbf{d}}$, one needs to estimate two sets of hyper-parameters and the scaling parameter $\rho$. This is done by using that the Gaussian processes $Z_{c}$ and $Z_{d}$ as independent of each other (i.e., $Z_{c}(\mathbf{x}) \perp Z_{d}(\mathbf{x})$ ) and finding maximum likelihood estimates (MLEs) for the associated means $\left(\mu_{c}\right.$ and $\left.\mu_{d}\right)$, variances $\left(\sigma_{c}^{2}\right.$ and $\left.\sigma_{d}^{2}\right)$, hyper-parameters $\left(\boldsymbol{\theta}_{c}\right.$ and $\left.\boldsymbol{\theta}_{d}\right)$ and the scaling parameter $\rho$. While analytical expressions exist for MLEs for the means and variances, one needs to maximize the two concentrated ln-likelihood functions numerically to solve for $\boldsymbol{\theta}_{c}, \boldsymbol{\theta}_{d}$ and $\rho$ (cf. Ref. [60] for details).

With hyper-parameters estimated, the co-kriging predictions of mean $\left(\mu_{e}^{\star}\right)$ and variance $\left(\sigma_{e}^{2^{\star}}\right)$ for the highfidelity model (i.e., the more expensive function), at a new point $\mathbf{x}^{\star}$ in the feature space, are

$$
\begin{aligned}
& \mu_{e}^{\star}=\hat{\mu}+\mathbf{k}^{\mathrm{T}} \mathbf{K}^{-1}(\mathbf{y}-\mathbf{1} \hat{\mu}) \\
& {\sigma_{e}^{2}}^{2^{\star}}=\hat{\rho}^{2}{\hat{\sigma_{c}}}^{2}+{\hat{\sigma_{d}}}^{2}-\mathbf{k}^{\mathrm{T}} \mathbf{K}^{-1} \mathbf{k}
\end{aligned}
$$

where, $\hat{\mu}=\mathbf{1}^{\mathrm{T}} \mathbf{K}^{-1} \mathbf{y} / \mathbf{1}^{\mathrm{T}} \mathbf{K}^{-1} \mathbf{1}, \mathbf{1}$ is a vector whose components are all one, and the symbol ${ }^{\wedge}$ is used over $\rho, \sigma_{c}$, and $\sigma_{d}$ to denote the MLEs of these parameters. The column vector $\mathbf{k}$ is given by

$$
\mathbf{k}=\left(\begin{array}{c}
\hat{\rho} \hat{\sigma}_{c}{ }^{2} \Im_{c}\left(\mathbf{X}_{\mathbf{c}}, \mathbf{x}^{\star}\right) \\
\hat{\rho}^{2}{\hat{\sigma_{c}}}^{2} \Im_{c}\left(\mathbf{X}_{\mathbf{e}}, \mathbf{x}^{\star}\right)+\hat{\sigma}_{d}^{2} \Im_{d}\left(\mathbf{X}_{\mathbf{e}}, \mathbf{x}^{\star}\right)
\end{array}\right)
$$

The notation $\Im_{c}\left(\mathbf{X}_{\mathbf{c}}, \mathbf{x}^{\star}\right)$, for instance, denotes a column vector of correlations between the data $\mathbf{X}_{\mathbf{c}}$ and the new point $\mathbf{x}^{\star}$ for which predictions are being made. It is also important to note that the framework discussed here assumes that there is no noise or uncertainty in the highfidelity data (i.e., the HSE06 bandgaps are being treated as the ground truth) and the Eq. 5 is an interpolator of the high-fidelity model (i.e., predictions at the known high-fidelity points will always coincide with the training values with vanishing variance).

$$
\Im\left(\mathbf{x}, \mathbf{x}^{\prime}, \boldsymbol{\theta}\right)=\exp \left\{-\sum_{j=1}^{d} \theta_{j}\left\|x_{j}-x_{j}^{\prime}\right\|^{2}\right\}
$$

When all the components $\theta_{j}$ are taken equal, the kernel is called isotropic. With the squared exponential correlation kernel, the resulting complete covariance matrix for the 2-level co-kriging model is
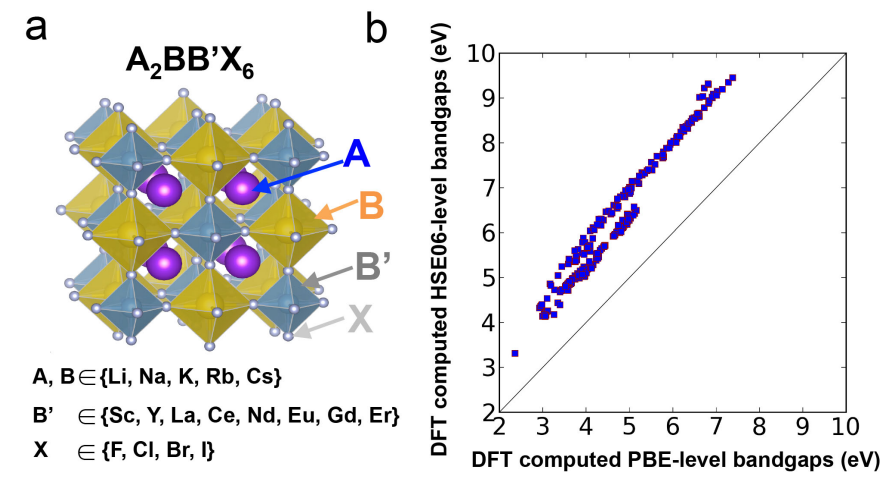

FIG. 2. (a) Crystal structure of $\mathrm{A}_{2} \mathrm{BB}^{\prime} \mathrm{X}_{6}$-type elpasolite compound. The octahedral environment of $\mathrm{B}$ - and $\mathrm{B}^{\prime}$-site cations is shown explicitly. The chemical space covered in the present study is also depicted. (b) Parity plot comparing the PBE and HSE06 bandgaps of 200 compounds used in the training and validation steps of the multi-fidelity ML model.

\section{Details of the Training Dataset}

To demonstrate the efficacy of the multi-fidelity bandgap learning model, we use a materials dataset of 640 double perovskite halides (or elpasolites) of $\mathrm{A}_{2} \mathrm{BB}^{\prime} \mathrm{X}_{6}$-type ( $c f$. Fig. 2a) employed in a recent study that was focused on identifying novel and improved scintillators - an application where the electronic bandgap is an important criterion for optimal performance. ${ }^{63}$ This materials database features halide compounds $(\mathrm{X}=\mathrm{F}$, $\mathrm{Cl}, \mathrm{Br}$ and I) with the A- and B- sites occupied by alkali metals $\mathrm{Li}, \mathrm{Na}, \mathrm{K}, \mathrm{Rb}$ and $\mathrm{Cs}$; the $\mathrm{B}^{\prime}$-site occupied by $\mathrm{Sc}$, Y, La, Ce, Nd, Eu, Gd and Er.

At the start, each of these 640 compounds were relaxed in a 20-atom $\sqrt{2} a_{c} \times \sqrt{2} a_{c} \times 2 a_{c}$ (with $a_{c}$ denoting a pseudo-cubic lattice parameter) supercell, using a guessed lattice parameter based on the empirical radii of the constituent atoms. ${ }^{64}$ Both lattice parameters as well as the internal coordinates were allowed to relax, using the PBE functional. ${ }^{19}$ We found that DFT 

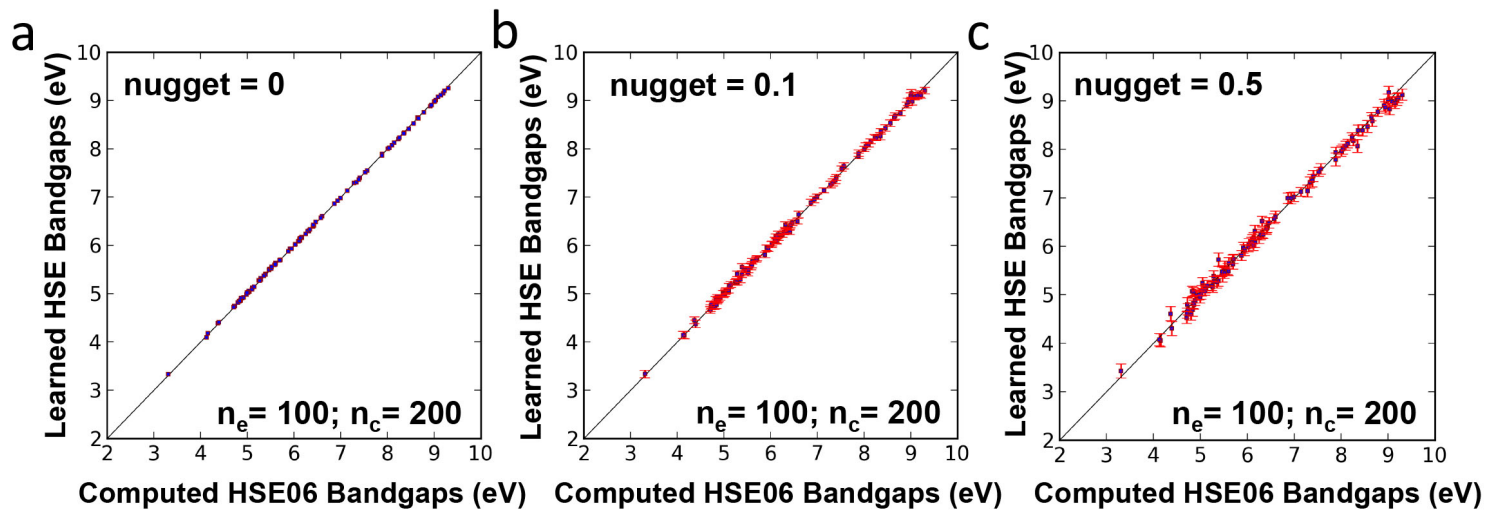

FIG. 3. Effect of the noise parameter (i.e., the nugget) on the ML models training performance. The 'learned' bandgaps (i.e., the mean values of the predictions) are compared with the bandgaps computed using HSE06 functional for a set of randomly selected 100 training compounds. The panels (a), (b) and (c) correspond to three different values of the nugget parameter and the error bars represent the root mean square error in the training.

PBE computations for a set of 41 compounds out of the total 640 systems failed to converge, even after repeated attempts, alluding towards unfeasibility of their synthetic realization. The PBE bandgaps of the remaining 599 compounds with converged electronic structure served as our low-fidelity bandgaps database. Next, a subset of 250 compounds from the low-fidelity bandgaps database was randomly selected, for which computations with the HSE06 functional ${ }^{17}$ were performed starting with the converged PBE-level electronic wavefunctions and relaxed geometry. The bandgaps dataset computed for these 250 compounds is henceforth referred to as the high-fidelity bandgap database. Out of these 250 compounds for which the HSE06 bandgaps are known from the DFT computations, we leave aside 50 compounds to begin with (which are never seen by the ML model in the training stage) and later use this data to evaluate model's predictive performance on unseen data. In combination with the PBE bandgaps, the HSE06 bandgaps of the remaining 200 compounds were used for training the multi-fidelity learning model.

To uniquely represent each compound as a feature vector $\left(\mathbf{x}_{c / e}^{(i)}\right)$ in Eq. 1, we use elemental features of the constituents atomic species occurring at the $\mathrm{A}, \mathrm{B}, \mathrm{B}^{\prime}$ and $\mathrm{X}$ sites of the compound. For each of the four atomic species, we use four elemental features (viz., electronegativity, and the first ionization potential, empirical radius and Pettifor's Mendeleev number) giving rise to a 16-dimensional feature vector to represent a compound in the target chemical space. The specific choice of these elemental features was based on previous studies in literature $26,54,55$ and our own recent work. ${ }^{58}$ Furthermore, owing to the symmetry with respect to swapping of the $\mathrm{B}$ and $\mathrm{B}^{\prime}$ cations in elpasolites, we use a convention in which we always arrange the $\mathrm{B}$ and $\mathrm{B}^{\prime}$ site cations in an increasing order of their atomic numbers, to uniquely identify each of the $\mathrm{A}_{2} \mathrm{BB}^{\prime} \mathrm{X}_{6}$ compounds.

\section{First Principles Computational Details}

In this work, we used first-principles DFT computations to optimize crystal structures and to compute the bandgaps in high-throughput manner. The DFT calculations were performed using projector-augmented wave formalism ${ }^{65,66}$ with the Perdew-Burke-Ernzerhoff $(\mathrm{PBE})^{19}$ functional as implemented in the Vienna $A b$ initio Simulation Package (VASP). ${ }^{67,68}$ The basis set included plane waves with kinetic energies up to $450 \mathrm{eV}$. For structural optimizations, a $\Gamma$-centered MonkhorstPack $k$-point mesh ${ }^{69}$ of $5 \times 5 \times 4$, giving the Kohn-Sham total energy converged up to $0.5 \mathrm{meV}$, was used. Relaxations of both the cell lattice vectors and internal atomic coordinates were allowed until atomic forces on each of the atoms were smaller than $0.01 \mathrm{eV} / \AA$. Spin unpolarized calculations were performed for all the systems. The high fidelity bandgaps for a set of randomly selected 250 compounds were computed using the hybrid HeydScuseria-Ernzerhof (HSE06) ${ }^{17}$ exchange-correlation functional, starting with the PBE-level relaxed structures and wave functions. The entire dataset of the computed PBE and HSE06 bandgaps is provided in the Supplementary Information.

\section{RESULTS AND DISCUSSIONS}

The parity plot presented in Fig. 2b compares our high-fidelity (HSE06) and low-fidelity (PBE) bandgap datasets for the 250-compound high-fidelity dataset. While, in general, a linear trend exists in the HSE06 and the PBE bandgaps, the bandgaps computed using the PBE functional are always underestimated as compared to the HSE06 bandgaps. Furthermore, the level of underestimation itself is dependent on the bandgaps (i.e., the larger the HSE06 bandgap, the larger is the difference between the HSE06 and PBE bandgaps). These 
a

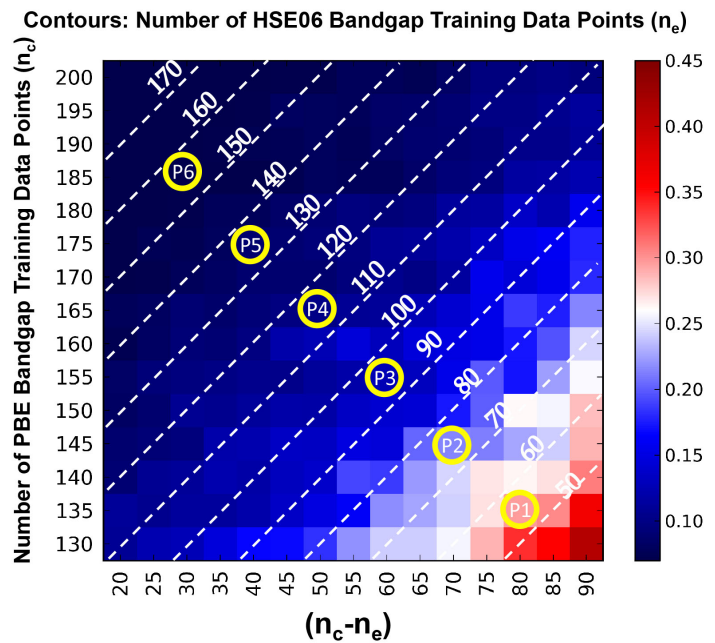

$\mathrm{b}$

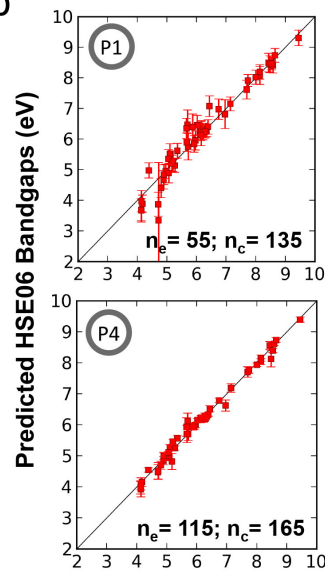

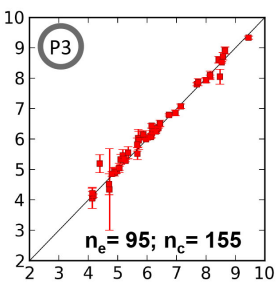
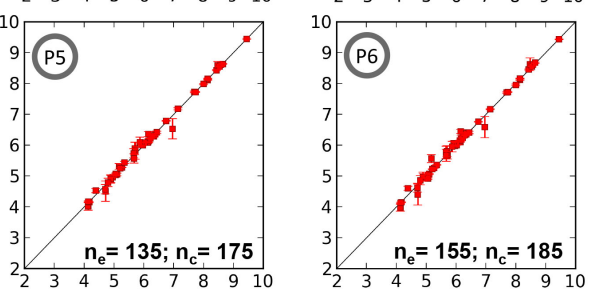

DFT Computed HSE06 Bandgaps (eV)

FIG. 4. (a) Learning performance of the multi-fidelity ML model as a function of number of low- and high-fidelity data points (i.e., $n_{c}$ and $n_{e}$, respectively) used in the training. Learning performance is evaluated as the MAE of the high-fidelity bandgap predictions and the DFT-computed HSE06 bandgaps on the validation set. To account for variability associated with the training set selection, for a given $n_{c}$ and $n_{e}$, an averaged MAE computed over 20 different ML models, each trained on a randomly selected training set is reported. (b) Panels P1 to P6 show representative parity plots between the high-fidelity bandgap predictions of the trained ML model and the DFT-computed HSE06 bandgaps on the validation set (i.e., a set of 50 left-out unseen data points) for selected pairs of $n_{c}$ and $n_{e}$. The panel labels are also appropriately indexed in (a).

trends are well known and are, in general, true when comparing the bandgaps computed within the local or semi-local density functionals with those computed using hybrid-functionals, beyond-DFT approaches (such as the quasi-particle GW approach) or experimental measurements.

Next, out of the 250 compositions shown in Fig. $2 \mathrm{~b}$ (for which both PBE- and HSE06-level bandgaps have been computed), we leave aside a set of 50 randomly selected compounds. We refer to this set as the validation set and neither the PBE nor HSE06 bandgaps of this set are used in model training. The validation set is used only to test the true predictive performance of our trained multi-fidelity ML model. Out of the remaining 200 chemistries, we randomly select $n_{c}$ compounds as our low-fidelity dataset (i.e., feature vectors $\mathbf{X}_{\mathbf{c}}$ and PBE bandgaps $\mathbf{y}_{\mathbf{c}}$ ) and $n_{e}$ compounds as our high-fidelity dataset (i.e., feature vectors $\mathbf{X}_{\mathbf{e}}$ and HSE06 bandgaps $\left.\mathbf{y}_{\mathbf{e}}\right)$. Also note here that the high-fidelity data is chosen such that $\mathbf{X}_{\mathbf{e}}$ is a subset of $\mathbf{X}_{\mathbf{c}}$; i.e. $\mathbf{X}_{\mathbf{e}} \subset \mathbf{X}_{\mathbf{c}}$.

Before moving onto the model's prediction performance, we first briefly comment about the learning performance of our multi-fidelity ML model. We note that the employed co-kriging model is an exact interpolator of the high-fidelity training data; that is, the trained model predicts exact values of the high-fidelity training data as mean given by Eq. 5, with a vanishing variance as per Eq. 6. This performance is easily rationalized by noting that while making predictions for an $i^{\text {th }}$ high-fidelity training data point $\mathbf{k}$ in Eq. 5 is the $n_{c}+i^{t h}$ column of $\mathbf{K}$ and therefore, the term $\mathbf{k}^{\mathrm{T}} \mathbf{K}^{-1}$ becomes the $n_{c}+i^{t h}$ unit vector leading to $\mu_{e}^{\star}=y_{e}^{(i)}$. Similarly, for Eq. 6 , the
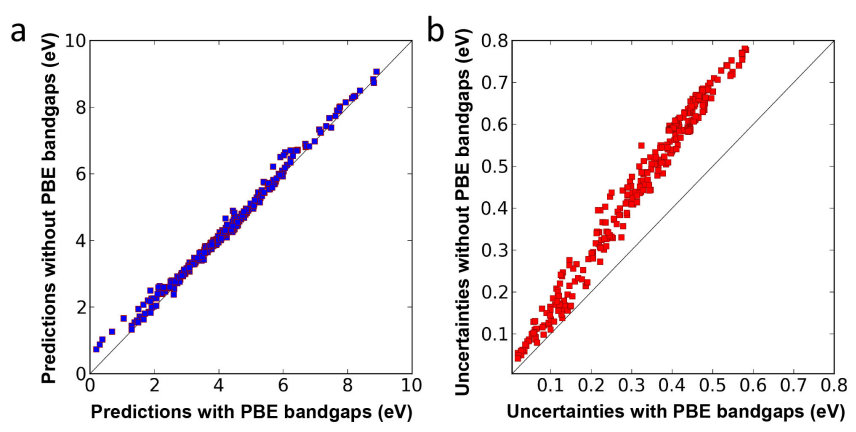

FIG. 5. A comparison between two sets of (a) means (i.e., $\mu_{e}{ }^{\star}$ in Eq. 5) and (b) standard deviations (i.e., $\sigma_{e}{ }^{\star}$ in Eq. 6) of ML-predicted bandgaps for the 349 elpasolite compounds not included in the training and validation sets. In the two sets, the predictions were made with and without including the PBE bandgaps of the compounds as low-fidelity data.

term $\mathbf{k}^{\mathrm{T}} \mathbf{K}^{-1} \mathbf{k}=\mathbf{k}^{\left(n_{c}+i\right)}$ becomes $\hat{\rho}^{2}{\hat{\sigma_{c}}}^{2}+{\hat{\sigma_{d}}}^{2}$, leading to a zero variance. This behavior is demonstrated in Fig. 3a, where we used $200 \mathrm{PBE}$ data points $\left(n_{c}=200\right)$ and a set of 100 randomly selected HSE06 data points $\left(n_{e}=100\right)$ from the training data set to train the multi-fidelity cokriging model. The presented parity plot shows that the trained ML model exactly predicts the values of the 100 HSE06 data points used in the training with vanishing variances.

It is also possible to modify the interpolating co-kriging formulation such that each analysis can be regressed appropriately to filter any measurement noise present in the training data. In the present context the measure- 


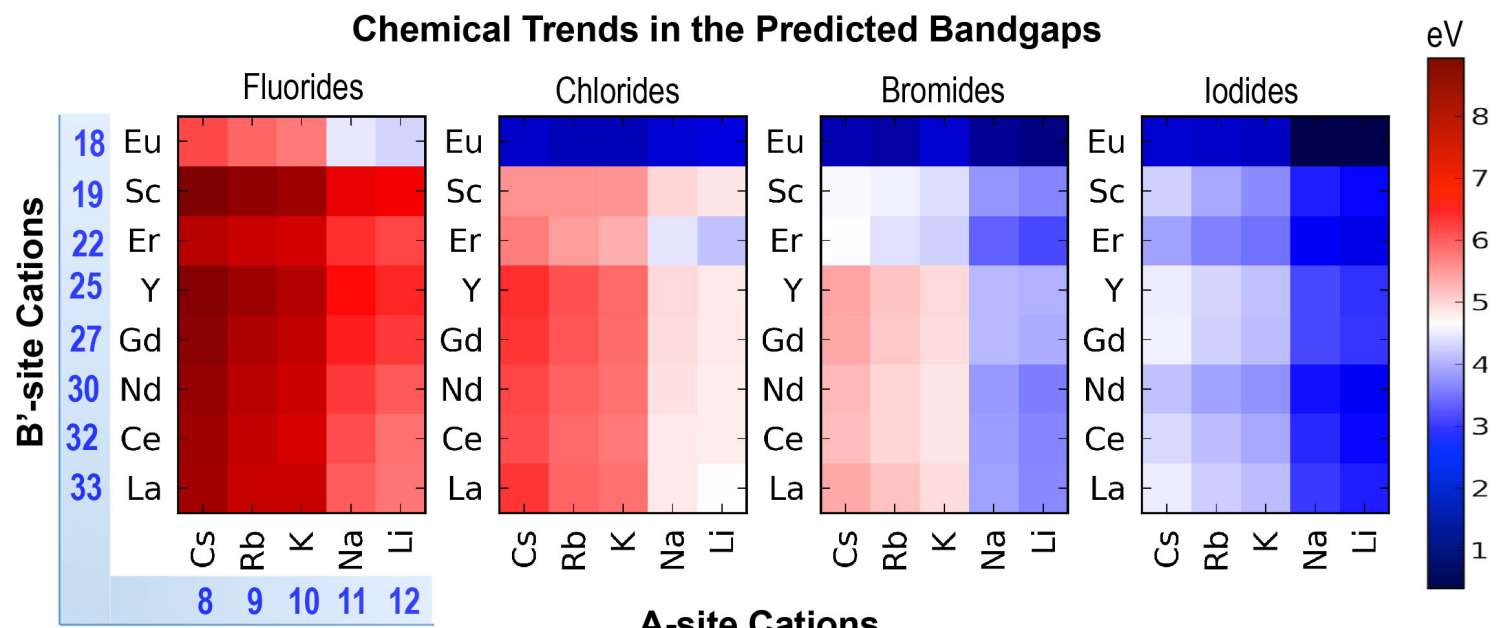

Pettifor's Mendeleev number

\section{A-site Cations}

FIG. 6. Analysis of chemical trends for the ML-predicted bandgap dataset. The four panels (from left to right) classify different $\mathrm{X}$-site chemistries for the elpasolite compounds. Each panel depicts an averaged predicted bandgap for a given A- and B'-site chemistry and averaged over all $\mathrm{A}^{\prime}$-site chemistries in the dataset. A- and $\mathrm{B}^{\prime}$-site elemental species are ordered according to the Pettifor's Mendeleev number.

ment noise can be thought of arising, for instance, from considering a finite discretization of the reciprocal space to compute the bandgaps. The incorporation of the measurement noise can easily be performed by adding a noise parameter (frequently referred to as the nugget) to the diagonal of associated the covariance matrices. ${ }^{60}$ While throughout this manuscript we focused on the noise-less case with the nugget parameter set to the machine precision, we did test the stability of our model with respect to the measurement noise for two different levels of nugget parameter set to 0.1 and 0.5 , as shown in Figs. 3b and $3 \mathrm{c}$ respectively. As seen from the figures, with a nonzero nugget parameter the trained model is not an exact interpolator of the high-fidelity training data and also exhibits non-zero variances for the data points. However, the predicted means still remain very close to the training data and the predicted variances are uniformly small, indicating the robustness of our trained model.

Figure 4a presents the high-fidelity bandgap prediction performance for a range of selected combinations of $n_{c}$ and $n_{e}$. The prediction performance is evaluated as the mean absolute error (MAE) on the validation set and the plotted MAEs represent an average performance evaluated over 20 different learning models, each with a given $n_{c}$ and $n_{e}$, but with different randomly selected training set compounds. It is clearly evident from the figure that as the number of low-fidelity or high-fidelity data points used in the training increases, the predictive performance of the model on unseen data also improves. Furthermore, the learning rate is relatively more sensitive to the number of high-fidelity data points used in the training process.

Figure 4b explicitly shows a systematic improvement in the predictive performance of the trained learning models with the number of training data points by directly com- paring the predicted (i.e., the mean as given by Eq. 5) and the DFT-computed bandgaps for the validation set. The error-bars on the predictions - representing the variance as given by Eq. 6 - are also plotted. Each of the parity plots (panels P1 through P6) corresponds to a fixed number of $n_{c}$ and $n_{e}$ data points (as indicted in the panels in Fig. 4b and also appropriately identified in the Fig. 4a). From Fig. 4b, it is seen that both the prediction performance as well as the uncertainties associated with the predictions systematically improve as both $n_{c}$ and $n_{e}$ gradually increase, going from panel P1 to $\mathrm{P} 6$.

After demonstrating the excellent prediction performance of the present ML model on unseen data using the left-out validation set, next we used the entire dataset (i.e., both the training and the validation set with the 250 HSE06 and the $250 \mathrm{PBE}$ data points) to train the multifidelity co-kriging model and predict HSE06 bandgaps of the remaining 349 compounds in the dataset. Since the PBE bandgaps of these compounds are also available, we can use the trained model to make predictions on the HSE06 bandgaps either directly or after incorporating the available PBE bandgaps as the low-fidelity data points. Figures $5 \mathrm{a}$ and $5 \mathrm{~b}$ compare the predicted means and variances, respectively, for the two sets of predictions made with and without including the PBE bandgaps of these 349 compounds. While the two sets of predicted means of the HSE06 bandgaps are, in general, in good agreement, incorporation of the additional PBE bandgap data points leads to significantly reduced variances in the predicted means, resulting in an improved model.

Finally, we analyze the predicted bandgaps to identify any systematic chemical trends that may be exhibited by various chemistries in the database. While the predicted bandgap values for the entire dataset are provided in the 
supplementary information, Fig. 6 captures the essence of our findings. In this figure, the four panels correspond to different X-site anions, dividing the compounds in the four groups of chemistries, viz. fluorides, chlorides, bromides and iodides. In each panel, the abscissa and the ordinate list the cations appearing at the $\mathrm{A}$ - and $\mathrm{B}^{\prime}$-sites of the elpasolites, respectively. Moreover, to better capture the underlying chemical trends, the cations are ordered according to the Pettifor's Mendeleev number an index that targets to capture the chemical similarity amongst different elemental species in the Periodic Table. The panels in Fig. 6 depict the predicted HSE06 bandgaps averaged over all chemistries with different Bsite cations that exist in the database. Therefore, while the dependence of the $\mathrm{A}$ - and $\mathrm{B}^{\prime}$-site chemistries with respect to the bandgap is explicitly represented by the plot, the variation with respect to the B-site cations is only captured in an averaged manner. The averaging over the B-site chemistries is justified since the bandgaps in the dataset only vary systematically and consistently with respect to the $\mathrm{B}$-site cations (i.e., for a given $\mathrm{A}$ - and $\mathrm{B}^{\prime}$-site cation, a larger B-site cation results in a smaller bandgap), therefore, allowing us to significantly simplify the analysis while still maintaining the overall chemical trends.

As seen from Fig. 6, as one moves from fluorides to iodides, the bandgaps systematically decrease with the fluorides exhibiting the largest bandgap values and the iodides the smallest. Also, we note that the overall relative trends with respect to the different $\mathrm{A}$ - and $\mathrm{B}^{\prime}$-site chemistries are still preserved in each of the four anionic subgroups. Furthermore, in contrast to our observation for the B-site, for a given X-site anion and B'-site cation, a larger A-site alkali metal cation (i.e., with a smaller Mendeleev number) leads to a larger bandgap. Finally, some trends along the $\mathrm{B}^{\prime}$-site cations can also be seen, though not as systematic as those observed for the Asite cations.

\section{CONCLUSIONS AND FUTURE OUTLOOK}

We presented and demonstrated the utility of a statistical learning method that combines low and high fidelity calculations of bandgaps in such a way to generate a machine-learned model that enables lower cost predictions of the bandgaps at the higher fidelity level. The presented approach is quite different from previous approaches using multi-fidelity data. ${ }^{15,26,54-58}$ We showed the method obviates the need for the exclusive use of high cost calculations for highly predictive models of bandgaps. We benchmarked the predictions of the method against actual high fidelity calculations of bandgaps of proposed materials. In all cases, the agreement between the exact and model calculations for elpasolites was excellent. Our predictions of bandgaps produced the expected chemical trends.

We comment that the use of the presented statistical learning method is not restricted to the predictions of bandgaps or is its use restricted to that with electronic structure methods. This method will be useful in many other predictions of materials parameters relevant to the search of new materials with enhanced functionalities when the cost between the use of computational methods with different levels of accuracy and cost is a factor. It implicitly extends the utility of low fidelity, high throughput calculations in a significant, novel way. Bandgaps and hierarchical DFT calculations provided convenient opportunities to demonstrate the approach.

Instead of being used to predict higher fidelities estimates of one targeted property, the method easily extends to predict multiple properties simultaneously. Used this way, it can provide valuable surrogates for material search problems where one is interested in identifying possible materials with multiple enhanced functionalities, for example, materials having both big bandgaps and large dielectric constants simultaneously. ${ }^{70,71}$ Multiple objective optimization is common to the search for new materials. The Gaussian process nature of the method also makes the method convenient for use in conjunction with methods based on adaptive materials design.

We note that multi-fidelity optimization is an active area of ML research. The ML method we used is relatively new. We also note that it is easily extended to other co-variance kernels besides Eq. 3. Additionally, it is possible to include estimates of the errors of the high accuracy calculations. While we studied two levels of accuracy, as we previously noted, using more than two is easily possible. The important issue, as with any ML method, is having a sufficient amount of statistically independent data to estimate the parameters in the model accurately. For the present method, it is also important that the data broadly sample the space of possible parameters to predict over the entire space accurately.

Acknowledgements: This paper is based on work supported by the U.S. Department of Energy through the LANL/LDRD program. G.P. would also like to gratefully acknowledge support from LANL's Director's postdoctoral fellowship program. Computational support for this work was provided by LANL's high performance computing clusters.

*Correspondence: Correspondence should be addressed to G.P. (email: gpilania@lanl.gov).
1 S. M. Sze and K. K. Ng, Physics of Semiconductor Devices, John Wiley \& Sons (2006).
2 A. Jain, Y. Shin, and K. A. Persson, Computational predictions of energy materials using density functional theory, 
Nat. Rev. Mater. 1, 15004 (2016).

3 R. Olivares-Amaya et al., Accelerated computational discovery of high-performance materials for organic photovoltaics by means of cheminformatics, Energy Environ. Sci. 4, 4849 (2011).

4 A. Nilsson, L. G. Pettersson, and J. Nørskov, Chemical bonding at surfaces and interfaces, Elsevier, Amsterdam, The Netherlands (2011)

5 M. J. Weber, Handbook of optical materials, CRC press, Boca Raton, USA (2002).

6 O. Madelung, Semiconductors: Data handbook, Springer Science \& Business Media (2012).

7 A. Mannodi-Kanakkithodi et al., Machine Learning Strategy for Accelerated Design of Polymer Dielectrics, Sci. Rep. 620952 (2016).

8 A. K. Singh, K. Mathew, H. L. Zhuang, and R. G. Hennig, Computational screening of $2 D$ materials for photocatalysis, J. Phys. Chem. Lett. 6, 1087 (2015).

${ }^{9}$ R. Gautier, X. Zhang, L. Hu, L. Yu, Y. Lin, T. O. Sunde, D. Chon, K. R. Poeppelmeier, and A. Zunger, Prediction and accelerated laboratory discovery of previously unknown 18-electron ABX compounds, Nat. Chem. 7, 308 (2015).

10 F. A. Rasmussen and K. S. Thygesen, Computational $2 D$ materials database: Electronic structure of transitionmetal dichalcogenides and oxides, J. Phys. Chem. C 119, 13169 (2015).

11 I. E. Castelli, K. S. Thygesen, and K. W. Jacobsen, Calculated optical absorption of different perovskite phases, J. Mater. Chem. A 3, 12343 (2015).

12 I. E. Castelli, D. D. Landis, K. S. Thygesen, S. Dahl, I. Chorkendorff, T. F. Jaramillo, and K. W. Jacobsen, Computational screening of perovskite metal oxides for optimal solar light capture, Energy Environ. Sci. 5, 5814 (2012).

13 I. E. Castelli, T. Olsen, S. Datta, D. D. Landis, S. Dahl, K. S. Thygesen, and K. W. Jacobsen, New cubic perovskites for one-and two-photon water splitting using the computational materials repository, Energy Environ. Sci. 5, 9034 (2012).

14 R. F. Berger and J. B. Neaton, Computational design of low-band-gap double perovskites, Phys. Rev. B 86, 165211 (2012).

15 W. Setyawan, R. M. Gaume, S. Lam, R. S. Feigelson, and S. Curtarolo, High-throughput combinatorial database of electronic band structures for inorganic scintillator materials, ACS Comb. Sci. 13, 382 (2011).

16 R. Armiento, B. Kozinsky, M. Fornari, and G. Ceder, Screening for high-performance piezoelectrics using highthroughput density functional theory, Phys. Rev. B 84, 014103 (2011).

17 J. Heyd, G. E. Scuseria, and M. Ernzerhof, Hybrid functionals based on a screened Coulomb potential, J. Chem. Phys. 118, 8207 (2003).

18 W. Kohn and L. J. Sham, Self-consistent equations including exchange and correlation effects, Phys. Rev. 140, A1133 (1965).

19 J. P. Perdew, K. Burke, and M. Ernzerhof, Generalized gradient approximation made simple, Phys. Rev. Lett. 77, 3865 (1996).

20 X. Ren, P. Rinke, C. Joas, and M. Scheffler, Random-phase approximation and its applications in computational chemistry and materials science, J. Mater. Sci. 47, 7447 (2012).

21 J. P. Perdew, A. Ruzsinszky, J. Tao, V. N. Staroverov, G. E. Scuseria, G. I. Csonka, Prescription for the design and selection of density functional approximations: More constraint satisfaction with fewer fits, J. Chem. Phys. 123, 062201 (2005).

${ }^{22}$ F. Aryasetiawan and O. Gunnarsson, The GW method, Rep. Prog. Phys. 61, 237 (1998).

${ }^{23}$ L. Hedin, New method for calculating the one-particle Green's function with application to the electron-gas problem, Phys. Rev. 139, A796 (1965).

24 C. Møller and M. S. Plesset, Note on an approximate treatment for many-electron systems, Phys. Rev. 46, 618 (1934).

25 J. A. Pople, M. Head-Gordon, and K. Raghavachari, Quadratic configuration interaction. A general technique for determining electron correlation energies, J. Chem. Phys. 87, 5968 (1987).

26 J. Lee, A. Seko, K. Shitara, K. Nakayama, and I. Tanaka, Prediction model of bandgap for inorganic compounds by combination of density functional theory calculations and machine learning techniques, Phys. Rev. B 93, 115104 (2016).

27 R. O. Jones, Density functional theory: Its origins, rise to prominence, and future, Rev. Mod. Phys. 87, 897 (2015).

28 A. Seidl, A. Görling, P. Vogl, J. A. Majewski, and M. Levy, Generalized Kohn-Sham schemes and the band-gap problem, Phys. Rev. B 53, 3764 (1996).

29 L. J. Sham and M. Schlüter, Density-functional theory of the energy gap, Phys. Rev. Lett. 51, 1888 (1983).

30 A. J. Cohen, P. Mori-Sánchez, and W. Yang, Fractional charge perspective on the bandgap in density-functional theory, Phys. Rev. B 77, 115123 (2008).

31 P. Mori-Sánchez, A. J. Cohen, and W. Yang, Localization and delocalization errors in density functional theory and implications for band-gap prediction, Phys. Rev. Lett. 100, 146401 (2008).

32 R. M. Martin, Electronic structure: Basic theory and practical methods, Cambridge University Press (2004).

33 J. P. Perdew and M. Levy, Physical content of the exact Kohn-Sham orbital energies: Bandgaps and derivative discontinuities, Phys. Rev. Lett. 51, 1884 (1983).

34 J. F. Janak, Proof that $\partial E / \partial n_{i}=\epsilon$ in density-functional theory, Phys. Rev. B 18, 7165 (1978).

35 V. I. Anisimov, F. Aryasetiawan, and A. I. Lichtenstein, First-principles calculations of the electronic structure and spectra of strongly correlated systems: the LDA+ U method, J. Phys. Condens. Matter 9, 767 (1997).

${ }^{36}$ F. Tran and P. Blaha, Accurate bandgaps of semiconductors and insulators with a semilocal exchange-correlation potential, Phys. Rev. Lett. 102, 226401 (2009).

37 J. Sun, A. Ruzsinszky, and J. P. Perdew, Strongly constrained and appropriately normed semilocal density functional, Phys. Rev. Lett. 115, 036402 (2015).

38 M. K. Y. Chan and G. Ceder, Efficient bandgap prediction for solids, Phys. Rev. Lett. 105, 196403 (2010).

39 F. Fuchs, J. Furthmüller, F. Bechstedt, M. Shishkin, and G. Kresse, Quasiparticle band structure based on a generalized Kohn-Sham scheme, Phys. Rev. B 76, 115109 (2007).

${ }^{40}$ K. Rajan, Materials informatics, Mater. Today 8, 38 (2005)

41 R. LeSar, Materials informatics: An emerging technology for materials development, Stat. Anal. Data Min. 1, 372 (2009).

42 T. Mueller, A. G. Kusne, and R. Ramprasad, Machine learning in materials science: Recent progress and emerging applications, A. L. Parrill and K. B. Lipkowitz, Eds., Reviews in Computational Chemistry, Wiley: New York, 
29, (2016).

43 M. Rupp, A. Tkathenko, K.-R. Muller, and O. A. von Lilienfeld, Fast and accurate modeling of molecular atomization energies with machine learning, Phys. Rev. Lett. 108, 058301 (2012).

${ }^{44}$ T. D. Huan, A. Mannodi-Kanakkithodi, and R. Ramprasad, Accelerated materials property predictions and design using motif-based fingerprints, Phys. Rev. B 92, 014106 (2015).

45 G. Pilania, C. Wang, X. Jiang, S. Rajasekaran, and R. Ramprasad, Accelerating materials property predictions using machine learning, Sci. Rep. 3, 2810 (2013).

46 V. Botu and R. Ramprasad, Adaptive machine learning framework to accelerate ab initio molecular dynamics, Int. J. Quantum Chem. 115, 1074 (2015).

47 V. Botu and R. Ramprasad, Learning scheme to predict atomic forces and accelerate materials simulations, Phys. Rev. B 92, 094306 (2015).

${ }^{48}$ L. M. Ghiringhelli, J. Vybiral, S. V. Levchenko, C. Draxl, and M. Scheffler, Big data of materials science: Critical role of the descriptor, Phys. Rev. Lett. 114, 105503 (2015).

49 G. Pilania, J. E. Gubernatis, and T. Lookman, Classification of octet AB-type binary compounds using dynamical charges: A materials informatics perspective, Sci. Rep. 5, 17504 (2015).

50 G. Pilania, P. V. Balachandran, J. E. Gubernatis, and T. Lookman, Classification of $\mathrm{ABO}_{3}$ perovskite solids: a machine learning study, Acta Crystallogr. Sect. B Struct. Sci. Cryst. Eng. Mater. 71, 507 (2015).

51 C. Kim, G. Pilania, and R. Ramprasad, From Organized High-Throughput Data to Phenomenological Theory using Machine Learning: The Example of Dielectric Breakdown, Chem. Mater. 28, 1304 (2016).

52 C. Kim, G. Pilania, and R. Ramprasad, Machine Learning Assisted Predictions of Intrinsic Dielectric Breakdown Strength of $\mathrm{ABX}_{3}$ Perovskites, J. Phys. Chem. C 120, 14575 (2016).

53 J. C. Snyder, M. Rupp, K. Hansen, K. R. Muller, and K. Burke, Finding density functionals with machine learning, Phys. Rev. Lett. 108, 253002 (2012).

54 P. Dey, J. Bible, S. Datta, S. Broderick, J. Jasinski, M. Sunkara, M.Menon, and K. Rajan, Informatics-aided bandgap engineering for solar materials, Comput. Mater. Sci. 83, 185 (2014).

55 T. Gu, W. Lu, X. Bao, and N. Chen, Using support vector regression for the prediction of the bandgap and melting point of binary and ternary compound semiconductors, Solid State Sci. 8, 129 (2006).

${ }^{56}$ G. Montavon, M. Rupp, V. Gobre, A. VazquezMayagoitia, K. Hansen, A. Tkatchenko, K.-R.Müller, and
O. A. von Lilienfeld, Machine learning of molecular electronic properties in chemical compound space, New J. Phys. 15, 095003 (2013).

57 R. Ramakrishnan, M. Hartmann, E. Tapavicza, and O. A. von Lilienfeld, Big data meets quantum chemistry approximations: The $\Delta$-machine learning approach, J. Chem. Theory Comput. 11, 2087 (2015).

58 G. Pilania, A. Mannodi-Kanakkithodi, B. P. Uberuaga, R. Ramprasad, J. E. Gubernatis, and T. Lookman, Machine learning bandgaps of double perovskites, Sci. Rep. 6, 19375 (2016).

59 M. C. Kennedy and A. O'Hagan, Predicting the output from a complex computer code when fast approximations are available, Biometrika 87, 1 (2000).

60 A. I. Forrester, A. Sóbester, and A. J. Keane, Multi-fidelity optimization via surrogate modelling, Proc. R. Soc. A 463, 3251 (2007).

${ }^{61}$ L. Le Gratiet, Bayesian analysis of hierarchical multifidelity codes, SIAM/ASA J. Uncertain. Quantif. 1, 244 (2013).

${ }^{62}$ L. Le Gratiet and J. Garnier, Recursive co-kriging model for design of computer experiments with multiple levels of fidelity, Int. J. Uncertain. Quantif. 4, 365 (2014).

63 F. P. Doty, P. Yang, and M. A. Rodriguez, Elpasolite scintillators, Sandia Natl. Lab2012 (2012).

64 J. C. Slater, Atomic radii in crystals, J. Chem. Phys. 41, 3199 (1964).

${ }^{65}$ P. E. Blöchl, Projector augmented-wave method, Phys. Rev. B 50, 17953 (1994).

66 G. Kresse and D. Joubert, From ultrasoft pseudopotentials to the projector augmented-wave method, Phys. Rev. B 59, 1758 (1999).

${ }^{67}$ G. Kresse and J. Furthmüller, Efficiency of ab-initio total energy calculations for metals and semiconductors using a plane-wave basis set, Comput. Mater. Sci. 6, 15 (1996).

68 G. Kresse and J. Furthmüller, Efficient iterative schemes for ab initio total-energy calculations using a plane-wave basis set, Phys. Rev. B 54, 11169 (1996).

69 H. J. Monkhorst and J. D. Pack, Special points for Brillouin-zone integrations, Phys. Rev. B 13, 5188 (1976).

70 A. Mannodi-Kanakkithodi, G. Pilania, and R. Ramprasad, Critical assessment of regression-based machine learning methods for polymer dielectrics, Comput. Mater. Sci. 125, 123 (2016).

71 A. Mannodi-Kanakkithodi, G. Pilania, R. Ramprasad, T. Lookman, and J. E. Gubernatis, Multi-objective optimization techniques to design the Pareto front of organic dielectric polymers, Comput. Mater. Sci. 125, 92 (2016). 